Pacific Journal of Mathematics

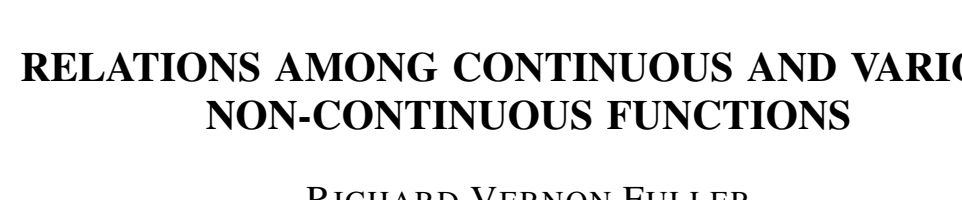




\title{
RELATIONS AMONG CONTINUOUS AND VARIOUS NON-CONTINUOUS FUNCTIONS
}

\author{
R. V. Fuller
}

In this paper a number of conditions on a function from one topological space to another are considered. Among these conditions are those of a function or its inverse preserving closedness, openness, or compactness of sets. Other conditions are having a closed graph and a concept generalizing continuity, subcontinuity, which we introduce.

Some interesting results which are uncovered are the following: (1) A function which is closed with closed point inverses and a regular space for its domain has a closed graph. (2) If a function maps into a Hausdorff space, continuity of the function is equivalent to the requirement that the function be subcontinuous and have a closed graph. (3) The usual net characterization of continuity for a function with values in a Hausdorff space is still valid if it is required only that the image of a convergent net be convergent (not necessarily to the "right" value).

Also several theorems of E. Halfar [2], [3] are extended including some sufficient conditions for continuity.

All spaces $X$ and $Y$ are topological spaces and no function is assumed to be continuous unless explicitly stated to be so. For any concepts which we do not define or elaborate upon, the reader is referred to Kelley's book [4]. We will denote nets by a symbol such as $x_{a}$, letting context distinguish between a net and a point of the range of the net and suppressing explicit mention of the directed set. A subnet of a net $x_{a}$ will be denoted by $x_{N b}$ where $b$ is a member of the domain of $x_{N b}$ and $N$ is the appropriate function from the domain of $x_{N b}$ to the domain of $x_{a}$.

1. Functions with closed graphs. A function $f: X \rightarrow Y$ has a closed graph (relative to $X \times Y$ ) if and only if $\{(x, f x): x \in X\}$ is closed in the product topology of $X \times Y$. Using a characterization of closed sets in terms of nets (see Kelley [4, Chapter 2)] a function $f: X \rightarrow Y$ has a closed graph if and only if $\left(x_{a}, f x_{a}\right)$ converges to $(p, q)$ in $X \times Y$ implies that $q=f p$. The well-known example of the differentiation operator from $C^{1}[0,1]$ to $C[0,1]$ shows that a function with a closed graph need not be continuous.

Let $E_{a}$ be a net of sets in a topological space, $X$. A point $p$ in $X$ belongs to $\lim \sup E_{a}$ (lim inf $E_{a}$ ) if and only if $E_{a}$ frequently (resp., eventually) intersects each neighborhood of $p$. This generali- 
zation of the lim sup and lim inf of sets from sequences to nets has been studied by Mrówka [8, p. 237].

LEMMA 1.1. If $f: X \rightarrow Y$ is a function, $y_{a}$ is a net in $Y$, and $p \in \lim \sup f^{-1}\left[y_{a}\right]$, then there is a net $x_{N b}$ in $X$ such that $x_{N b} \rightarrow p$ $\left(x_{N b}\right.$ converges to $\left.p\right)$ and $f x_{N b}$ is a subnet of $y_{a}$.

Proof. Assume $y_{a}$ is a net in $Y$ and $p \in \lim \sup f^{-1}\left[y_{a}\right]$. Then for each index $a$ and each neighborhood of $p, U$, there is an index $N(a, U) \geqq a$ such that $f^{-1}\left[y_{N(a, U)}\right] \cdot U \neq \varphi$. Now direct the neighborhoods $U$ downward by inclusion, give the pairs $(a, U)$ the product direction, and choose $x_{N(a, U)} \in f^{-1}\left[y_{N(a, U)}\right] \cdot U$, thus obtaining a net $x_{N(a, U)}$. Finally note that $f x_{N(a, U)}=y_{N(a, U)}$ is a subnet of $y_{a}$ and $x_{N(a, U)} \rightarrow p$.

The following characterization of a function with a closed graph appears in Kuratowski [5, Defn., p. 32] in a considerably restricted form.

Theorem 1.2. If $f: X \rightarrow Y$ is a function, the following conditions are equivalent:

(a) The function $f$ has a closed graph.

(b) If $y_{a} \rightarrow q$ in $Y$, then $\lim \sup f^{-1}\left[y_{a}\right] \subset f^{-1}[q]$.

(c) If $y_{a} \rightarrow q$ in $Y$, then $\liminf f^{-1}\left[y_{a}\right] \subset f^{-1}[q]$.

Proof. Assume (a) holds. Let $y_{a} \rightarrow q$ in $Y$ and $q \in \lim \sup f^{-1}\left[y_{a}\right]$. By Lemma 1.1 there is a net $x_{N b}$ in $X$ such that $x_{N b} \rightarrow p$ and $f x_{N b}$ is a subnet of $y_{a}$. Thus we have $\left(x_{N b}, f x_{N b}\right) \rightarrow(p, q)$. Since $f$ has a closed graph $q=f p$ or $p \in f^{-1}[q]$. Consequently $(b)$ holds.

If (b) holds then, since $\liminf f^{-1}\left[y_{a}\right]$ is a subset of $\lim \sup f^{-1}\left[y_{a}\right]$, (c) evidently holds.

Assume then that (c) holds. Suppose $\left(x_{a}, f x_{a}\right) \rightarrow(p, q)$. Then $y_{a}=f x_{a} \rightarrow q$ and $p \in \liminf f^{-1}\left[y_{a}\right]$. Therefore $p \in f^{-1}[q]$ or $q=f p$ and (a) holds.

2. Subcontinuous and inversely subcontinuous functions. The function $f: X \rightarrow Y$ is said to be subcontinuous if and only if $x_{a} \rightarrow p$ in $X$ implies there is a subnet of $f x_{a}, f x_{N b}$, which converges to some point $q$ in $Y$. Similarly a function $f: X \rightarrow Y$ is said to be inversely subcontinuous if and only if $f x_{a} \rightarrow q$ in $Y$ implies there is a subnet of $x_{a}, x_{N b}$ which converges to some point $p$ in $X$, that is, if and only if $y_{a} \rightarrow q$ in $Y$ and $x_{a} \in f^{-1}\left[y_{a}\right]$ implies that a subnet of $x_{a}$, $x_{N b}$, converges to some point $p$ in $X$.

Our concept of a subcontinuous (inversely subcontinuous) function is a generalization of a function whose range (resp., domain) is compact. In addition, a subcontinuous function is a generalization of a 
continuous function whence its name.

More generally it is clear that if $f: X \rightarrow Y$ is function and each point $p$ in $X$ (in $Y$ ) has a neighborhood $U$ such that $f[U]$ (resp., $\left.f^{-1}[U]\right)$ is contained in a compact subset of $Y($ resp., of $X)$ then $f$ is subcontinuous (resp. inversely subcontinuous).

There are a couple more analogous pairs of facts about subcontinuous and inversely subcontinuous functions with analogous proofs of these facts. This seems to suggest that each pair of proofs could have been integrated into a single proof concerning a multiple-valued subcontinuous function. However such an approach seemed somewhat astray from the present study.

The following theorem says that a subcontinuous (inversely subcontinuous) function $f: X \rightarrow Y$ where $Y$ (resp. $X$ ) is completely regular is very nearly such that $f$ (resp., $f^{-1}$ ) preserves compactness. Unfortunately nearly is often not good enough. However subcontinuous (inversely subcontinuous) functions have advantages over those such that the function (resp., the inverse) preserves compactness as will be seen later.

TheOREM 2.1. Let $f: X \rightarrow Y$ be a function. If $f$ is subcontinuous (inversely subcontinous) and $Y$ (resp., $X)$ is completely regular then for each compact subset of $X$ (resp., $Y), K, f[K]^{-}\left(\right.$resp. $\left.f^{-1}[K]^{-}\right)$ is compact. (-denotes closure).

Proof. We prove only the assertions for $f$ inversely subcontinuous, the proof for $f$ subcontinuous being entirely analogous.

Let $K$ be a compact subset of $Y$. Let $\left\{z_{a}, a \in A\right\}$ be a net in $f^{-1}[K]^{-}$. Let $B$ be a uniformity for $X$. Direct $B$ downward by inclusion and let $A \times B$ have the product order. For each $(a, b) \in A \times B$ choose $x_{(a, b)}$ in $b\left[z_{a}\right] \cdot f^{-1}[K]$. Since $K$ is compact, a subnet of $f x_{(a, b)}$ converges and thus a subnet $x_{(N c, M c)}$ of $x_{(a, b)}$ converges to some $p$ in $X$. Clearly $p \in f^{-1}[K]^{-}$.

Now consider the net $z_{N o}$ which is a subnet of $z_{a}$. We proceed to show $z_{N c} \rightarrow p$. Let $\mathrm{b} \in B$. There is a symmetric $b_{1} \in B$ such that $b_{1} \circ b_{1} \subset b$. By choice of the $x_{(a, b)}$, it is clear that $\left(z_{N c}, x_{(N c, M c)}\right)$ is eventually in $b_{1}$. Since $x_{(N c, M c)} \rightarrow p,\left(x_{(N c, M c)}, p\right)$ is eventually in $b_{1}$. Thus $\left(z_{N c}, p\right)$ is eventually in $B$ and $z_{N c} \rightarrow p$. Consequently $f^{-1}[K]^{-}$is compact.

3. Functions and inverses which preserve closedness and compactness. Let $f: X \rightarrow Y$ be a function. If $f\left(f^{-1}\right)$ takes compact sets of $X$ (resp., $Y$ ) onto compact sets of $Y$ (resp., $X$ ) then $f$ is said to be compact-preserving (resp., compact). If $f\left(f^{-1}\right)$ takes closed sets of $X$ (resp., $Y$ ) onto closed sets of $Y$ (resp., $X$ ) then $f$ is said to be 
closed (resp., continuous).

Pursuing further the similarities noted in the previous section, we characterize compact and compact preserving functions in terms of inverse subcontinuity and subcontinuity, respectively.

THEOREM 3.1. Let $f: X \rightarrow Y$ be a function.

(a) The function $f$ is compact if and only if $f \mid f^{-1}[K]: f^{-1}[K] \rightarrow K$ is inversely subcontinuous for each compact set $K \subset Y$.

(b) The function $f$ is compact-preserving if and only if $f \mid K: K \rightarrow f[K]$ is subcontinuous for each compact set $K \subset X$.

Proof. The proof of (b) is entirely analogous to that of (a) and so only (a) is proved.

Assume $f$ is compact. Let $K$ be a compact subset of $Y$ and $f x_{a}$ a net in $K$ which converges to $q$ in $K$. Then $x_{a}$, being in the compact set $f^{-1}[K]$, has a subnet $x_{N b}$ converging to some $p$ in $f^{-1}[K]$. Thus $f \mid f^{-1}[K]: f^{-1}[K] \rightarrow K$ is inversely subcontinuous.

Now assume the subcontinuity condition. Let $K$ be a compact subset of $Y$ and let $x_{a}$ be a net in $f^{-1}[K]$. Then $f x_{a}$ has a subnet converging to a point in $K$ and thus using the condition, $x_{a}$ has a subnet converging to a point in $f^{-1}[K]$. Therefore $f^{-1}[K]$ is compact.

The following theorem giving sufficient conditions that a function be compact or compact preserving is deduced immediately from Theorem 3.1.

THEOREM 3.2. Let $f: X \rightarrow Y$ be a function. If $f$ is subcontinuous (inversely subcontinuous) and $f[K]\left(\right.$ resp., $\left.f^{-1}[K]\right)$ is closed for each compact subset, $K$, of $X$ (resp., $Y$ ) then $f$ is compact preserving (resp., compact).

From Theorem 3.6 it will then follow that $f$ has a closed graph and is subcontinuous (inversely subcontinuous) implies $f$ is compactpreserving (resp., compact).

Corollary 3.3. Let $f: X \rightarrow Y$ be a function. If $X(Y)$ is Hausdorff and $f$ is both closed and subcontinuous (resp., continuous and inversely subcontinuous) then $f$ is compact-preserving (resp., compact).

We turn now to gathering some more facts about functions with closed graphs and their relation to other functions. In particular, the following two theorems show that the closed graph property complements the two subcontinuities in interesting ways.

Theorem 3.4. Let $f: X \rightarrow Y$ be a function. A sufficient condition that $f$ be continuous is that $f$ have a closed graph and be subcontinuous. If $Y$ is Hausdorff, the condition is also necessary. 
Proof. (Sufficiency) Let $x_{a}$ be a net in $X$ which converges to some point $p$. Suppose $f x_{a}$ does not converge to $f p$. Then $f x_{a}$ has a subnet, $f x_{N b}$, no subnet of which converges to $f p$. However, by subcontinuity, some subnet of $f x_{N b}, f_{N M c}$, converges to some point $q$. Thus we have $\left(x_{M N c}, f x_{N M c}\right) \rightarrow(p, q)$. But by the closed graph property of $f, q=f p$ and we have a contradiction.

(Necessity) Assuming $f$ is continuous then evidently $f$ is subcontinuous. If $\left(x_{a}, f x_{a}\right) \rightarrow(p, q)$ then $x_{a} \rightarrow p$ and thus $f x_{a} \rightarrow f p$. Assuming $Y$ is Hausdorff we conclude that $q=f p$ and $f$ accordingly has a closed graph.

THeOREM 3.5. If the function $f: X \rightarrow Y$ has a closed graph and is inversely subcontinuous, then $f$ is closed.

Proof. Let $C$ be a closed subset of $X$. Suppose $f[C]$ is not closed. Then there is a $q$ in $Y-f[C]$ and a net $x_{a}$ in $C$ such that $f x_{a} \rightarrow q$. Thus by inverse subcontinuity there is a subnet of $x_{a}, x_{N b}$, which converges to some $p$ in $X$. Since $C$ is closed $p \in C$. Consequently we have $\left(x_{N b}, f x_{N b}\right) \rightarrow(p, q)$, but $q \neq f p$ since $q \notin f[C]$. This contradicts the closedness of the graph of $f$.

Theorem 3.4 tells us that if $f: X \rightarrow Y$ is continuous with $Y$ Hausdorff then $f$ has a closed graph. The Hausdorff requirement cannot be dropped in general for if $i: X \rightarrow X$ is the identity on $X$, the graph of $i$ is closed in $X \times X$ if and only if $X$ is Hausdorff.

We can also see from Theorem 3.4 the dependency of the closedgraphness of a function $f: X \rightarrow Y$ upon the space $Y$ in which $f[X]$ is imbedded.

Let $f: X \rightarrow Y$ be a function which has a closed graph and is not continuous. Let $Y^{*}$ be a compactification of $Y$. Then $f: X \rightarrow Y^{*}$ is subcontinuous (showing also the dependency of subcontinuity on the space in which the range is embedded). Thus if $f: X \rightarrow Y^{*}$ had a closed graph then it would be continuous. But continuity does not depend upon the embedding space for the range so that $f: X \rightarrow Y$ would be continuous contradicting our assumption.

Assuming the range is embedded in a Hausdorff space, the next theorem says, roughly, that a function with a closed graph handles compact sets somewhat less successfully than a continuous function but its inverse does just as well as the inverse of a continuous functions in the treatment of compact sets. The theorem follows from an exercise in Kelley [4, Ex. A, p. 203] but we also include a proof.

THEOREM 3.6. Let the function $f: X \rightarrow Y$ have a closed graph. If $K$ is a compact subset of $X(Y)$ then $f[K]$ (resp., $\left.f^{-1}[K]\right)$ is a closed 
subset of $Y($ resp., $X)$.

Proof. We prove only the second case, the proof of the first being entirely analogous.

Let $K$ be a compact subset of $Y$. Suppose $f^{-1}[K]$ is not closed. Then there is a $p$ in $X-f^{-1}[K]$ and a net $x_{a}$ in $f^{-1}[K]$ such that $x_{a} \rightarrow p$. Evidently $f x_{a}$ has a subnet $f x_{N b}$ which converges to some $q$ in $K$. Thus we have $\left(x_{N b}, f x_{N b}\right) \rightarrow(p, q)$ so that $p \in f^{-1}[q] \subset f^{-1}[K]$. But this contradicts the choice of $p$.

Having already discovered in Theorem 3.4 conditions under which a continuous function has a closed graph, we proceed to find such conditions for a closed function. The characteristic function of the interval $(0,1]$ mapping $E_{1}$ into $\{0,1\}$ shows us that a closed function does not always have a closed graph. One should note that this function does not have closed point inverses.

Let us call a function $f: X \rightarrow Y$ locally closed if for every neighborhood, $U$, of each point $p$ in $X$, there is a neighborhood of $p, V$, such that $V \subset U$ and $f[V]$ is closed in $Y$ (Whyburn [12, p. 198] has used the same term differently). It is not clear that a closed function is always locally closed but if the domain of a closed function is regular, then the function is locally closed. Also if a function $f: X \rightarrow Y$ is such that $X$ is regular and locally compact and $f$ takes compact sets onto closed sets, then $f$ is locally closed.

A locally closed function need not be closed as the following example shows. Let $X$ be the reals with the discrete topology, $Y$ the reals with the usual topology, and $f: X \rightarrow Y$ the identity function. Then $f$ is locally closed and, in fact, continuous but certainly not closed.

THEOREM 3.7. If $f: X \rightarrow Y$ is a locally closed function then $y_{a} \rightarrow q$ in $Y$ implies $\lim \sup f^{-1}\left[y_{a}\right] \subset f^{-1}[q]^{-}$.

Proof. Let $y_{a} \rightarrow q$ in $Y$ and $p \in \lim \sup f^{-1}\left[y_{a}\right]$. Suppose $p \notin f^{-1}[q]^{-}$. By Lemma 1.1 there is a net $x_{N b}$ in $X$ such that $x_{N b} \rightarrow p$ and $f x_{N b}$ is a subnet of $y_{a}$. Now $X-f^{-1}[q]^{-}$is a neighborhood of $p$ and thus there is a neighborhood of $p, U$, such that $U \subset X-f^{-1}[q]^{-}$and $f[U]$ is closed in $Y$. But $x_{N b}$ is eventually in $U$ so that $f x_{N b}$ is eventually in $f[U]$. This means $f x_{N b}$ is eventually in the complement of a neighborhood of $q$, namely $Y-f[U]$. We therefore have a contradiction to the fact that $f x_{N b}$ must converge to $q$, being a subnet of $y_{a}$.

CoROLlaRY 3.8. If $f: X \rightarrow Y$ is a locally closed function and has closed point inverses then $f$ has a closed graph. 
Proof. The statement follows immediatelly from Theorem 1.2.

CoRollary 3.9. If the function $f: X \rightarrow Y$ is closed with closed point inverses and $X$ is regular then $f$ has a closed graph.

CoROLlary 3.10. If the function $f: X \rightarrow Y$ is closed and subcontinuous with closed point inverses and $X$ is regular then $f$ is continuous.

Proof. We have this result directly from 3.9 and Theorem 3.4.

The last corollary generalizes a theorem of Halfar's [2, Th. 3] by replacing compactness of $Y$ with subcontinuity of $f$. See also in this connection Rhoda Manning [6, Th. 1.5].

THEOREM 3.11. If $f: X \rightarrow Y$ is a function where $X$ is regular and locally compact, the following conditions are equivalent:

(a) $f$ maps compact sets onto closed sets and has closed point inverses.

(b) $f$ is locally closed and has closed point inverses.

(c) $f$ has a closed graph.

Proof. We have already commented that (a) implies (b). By Corollary 3.8, (b) implies (c). Thus it remains to show (c) implies (a). Assuming $f$ has a closed graph, Theorem 3.6 gives us that $f$ maps compact sets onto closed sets. Furthermore since points are compact, the same theorem yields that $f$ has closed point inverses.

The following theorem, which should be compared with Theorem 3.2 , is essentially a special case of one to be found in Bergé [1. Th. 3, p. 116]. The theorem extends one of Halfar's [2, Th. 1] by removing the requirement that $f$ be continuous. Also of interest in this connection are Michael [7, Lemma 5.18, p. 172] and Whyburn [11, Th. 3 \& Cor. 2, pp. 689-690].

THEOREM 3.12. If $f: X \rightarrow Y$ is closed and has compact point inverses, then $f$ is compact.

4. Compact functions, compact-preserving functions, and $k_{i}$ spaces. It is intuitively clear that a mapping $f: X \rightarrow Y$ which is compact or compact preserving will have no particular tendency to treat other topological properties nicely unless the topologies of $X$ or $Y$ or both are to a considerable extent dictated by the compact sets. In this section we define some topological spaces which are, in a sense, determined by their compact sets, and prove a couple of theorems concerning these spaces and compact preserving functions. 
Let $X$ be a topological space and $p \in X . \quad X$ is said to have property $k_{1}$ at $p$ and only if for each infinite subset $A$ having $p$ as an accumulation point, there is a compact subset of $A+p, B$, such that $p \in B$ and $p$ is an accumulation point of $B . \quad X$ is a $k_{1}$ space if it has property $k_{1}$ at each of its points.

$X$ has property $k_{2}$ at $p$ if and only if for each set $A$ having $p$ as an accumulation point, there is a subset of $A, B$, and a compact set $K \supset B+p$ such that $p$ is an accumulation point of $B$. We call $X$ a $k_{2}$ space if it has property $k_{2}$ at each of its points.

$X$ is a $k_{3}$ space if and only if $U$ is an open set in $X$ precisely whenever $U \cdot K$ is open in $K$ for each compact set $K$ in $X$.

Halfar defines property $k_{1}$ at a point in one of his papers 13, Defn. 2]. Property $k_{2}$ at a point is a slight variation of a definition I believe is due to S. B. Myers. A definition differing slightly from that of a $k_{3}$ space is discussed by Kelley in his book [4, p. 230]. If $X$ is Hausdorff, the $k_{2}$ and $k_{3}$ definitions agree with those of Myers and Kelley respectively.

It is immediate that $X$ is $k_{1}$ at $p$ implies $X$ is $k_{2}$ at $p$. Also it is not difficult to show that a $k_{2}$ space is always a $k_{3}$ space, but I do not know whether $k_{2}$ space and $k_{3}$ space are equivalent concepts. It is easy to see that a locally compact or first countable space is a $k_{2}$ space. Finally the following example shows that $X$ being $k_{2}$ at a point $p$ does not necessitate $X$ being $k_{1}$ at $p$.

EXAMPle 4.1. This example provides a $k_{2}$ space which does not have property $k_{1}$ at any point.

Let $F$ be the space of all functions mapping $[0,1]$ into $[0,1]$ with the topology of point-wise convergence. Let $\mathscr{A}$ be the collection of all finite subsets of $[0,1]$ and $\omega$ the set of positive integers. For each $A$ in $\mathscr{A}$ and $n$ in $\omega$ let $f_{n A}$ be the function in $F$ defined by $f_{n A} x=1 / n$ for $x$ in $A$ and $f_{n A} x=1$ otherwise. Letting $\mathscr{A}$ be directed upward by inclusion, $\omega$ have the usual order, and $\omega \times \mathscr{C}$ have the product order, then $\left\{f_{n A},(n, A) \in \omega \times \mathscr{A}\right\}$ is a net in $F$.

It is easy to see that $f_{n A}$ converges to the zero function, $0^{*}$, and thus that $0^{*}$ is an accumulation point of $\left\{f_{n A}:(n, A) \in \omega \times \mathscr{D}\right\}$. We proceed to show $F$ lacks property $k_{1}$ at $0^{*}$ and the proof that $F$ has property $k_{1}$ at no point will then be clear.

Let $p=\left\{f_{n A}:(n, A) \in \omega \times \mathscr{C}\right\}$ and let $B$ be any subset of $P$ which has $0^{*}$ as an accumulation point. Then $\left.\{n, A): f_{n A} \in B\right\}$ must be cofinal in $\omega \times \mathscr{A}$ for if for any $\left(n_{0}, A_{0}\right)$ there is no $\left(n_{1}, A_{1}\right) \geqq\left(n_{0}, A_{0}\right)$ such that $f_{n_{1} A_{1}} \in B$ then there is no member of $B$ in the neighborhood of $0^{*}$ given by $\left\{f \in F:|f x|<1 / n_{0}\right.$ for $\left.x \in A_{0}\right\}$.

Now let $f_{n_{0} A_{0}} \in B$. For every $k$ in $\omega$ choose $f_{n_{k} A_{k}} \in B$ such that $n_{k}>n_{k-1}$ and $A_{k} \supset A_{k-1}$. Consider $Q=\Sigma\left\{A_{k}: k \in \omega\right\}$. Let $f \in F$ be 
defined by $f x=0$ for $x$ in $Q, f x=1$ otherwise. The sequence $f_{n_{k} A_{k}}$ converges to $f$ and $f \neq 0^{*}, 0, f \notin P$ since $Q$ is countable. Thus $B+0^{*}$ is not closed and since $F$ is Hausdorff it is consequently not compact.

We conclude $F$ does not have property $k_{1}$ at any point, but on the other hand since by the Tychonoff theorem $F$ is compact, it is clearly a $k_{2}$ space.

The next theorem extends one of Halfar [2, Th. 2] by requiring that $f$ have only a closed graph instead of being continuous and by requiring that $Y$ only be a $k_{3}$ space instead of locally compact Hausdorff. The theorem should be compared with a similar one in Whyburn $[11$, p. 690].

THEOREM 4.2. Let $f: X \rightarrow Y$ be a compact function and $Y$ a $k_{3}$ space. A sufficient condition that $f$ be closed is that $f$ have a closed graph. If $X$ is regular Hausdorff, the condition is also necessary.

Proof. Assume $f$ has a closed graph. Let $C$ be a closed subset of $X$. Since $Y$ is a $k_{3}$ space, to show that $f[C]$ is closed we have only to show that the intersection of $f[C]$ with each compact set $Y$, $K$, is closed in $K$.

Let $K$ be a compact subset of $Y$. Then $f^{-1}[K]$ is compact, and it follows that $C \cdot f^{-1}[K]$ is compact. By Theorem 3.6 we have that $f\left[C \cdot f^{-1}[K]\right]$ is closed. Finally since

$$
f\left[C \cdot f^{-1}[K]\right]=f[C] \cdot K
$$

$f[C] \cdot K$ is closed in $Y$ and thus in $K$.

Now assume that $X$ is regular Hausdorff and $f$ is closed. Then $f$ being compact, point inverses are closed. Thus by Corollary $3.9, f$ has a closed graph.

Comparing Theorem 3.5 and the sufficiency part of the one immediately preceding, we see that in the former theorem " $f$ is inversely subcontinuous" replaces " $f$ is compact" and no requirements are put on the space $Y$. The example which follows shows that the requirement that $Y$ be a $k_{3}$ space cannot be dropped in Theorem 4.3. These observations illustrate that in some instances $f$ being inversely subcontinuous is effectively a stronger requirement than $f$ being compact.

EXAMPLE 4.3. We will display a function which is compact and has a closed graph but which is not closed.

Let $X$ be an uncountable set. Let $\mathscr{U}_{1}$ be the topology for $X$ consisting of all compliments of countable sets (plus the empty set). Let $\mathscr{C}_{2}$ be the discrete topology on $X$. It is known that the only 
compact subsets of either $\left(X, \mathscr{C}_{1}\right)$ or $\left(X, \mathscr{C}_{2}\right)$ are the finite sets.

Consider the identity map $i:\left(X, \mathscr{C}_{2}\right) \rightarrow\left(X, \mathscr{C}_{1}\right)$. The function $i$ has a closed graph as we now show. Let $\left(x_{a}, i x_{a}\right) \rightarrow(p, q)$. Then $x_{a}$ must eventually be the constant $p$ in $\left(X, \mathscr{C}_{1}\right)$ and so also must $i x_{a}=x_{a}$. If $i x_{a}$ converged to $q \neq q$ then we would have a contradiction to the fact the points are closed in $\left(X, \mathscr{C}_{1}\right)$.

The inverse of $i$ carries compact (finite) subsets of $\left(X, \mathscr{C}_{1}\right)$ onto compact (finite) subsets of $\left(X, \mathscr{C}_{2}\right)$ and $i$ is consequently compact. Finally since $\mathscr{U}_{2}$ is a strictly large topology than $\mathscr{U}_{1} i$ is not closed.

THEOREM 4.4. Let $f: X \rightarrow Y$ be a function where $X$ and $Y$ are Hausdorff and $X$ has property $k_{2}$ at a point $p$. If $f$ is compact preserving and has closed point inverses, then $f$ is continuous at $p$.

Proof. Suppose $f$ is not continuous at $p$. Then there is a neighborhood $V^{*}$ of $f p$ such that for each neighborhood of $p, U$, there exists a point $x_{U}$ in $U$ with the property that $f x_{U} \notin V^{*}$. The collection $A=\left\{x_{U}: U\right.$ is a neighborhood of $\left.p\right\}$ has $p$ as our accumulation point. Thus $A$ has a subset $B$ and a compact set $K \supset B+p$ such that $p$ is an accumulation point of $B$.

Consider the function $f \mid K: K \rightarrow Y$. The function $f \mid K$ is (strongly) closed since $Y$ is Hausdorff. As

$$
(f \mid K)^{-1}[p]=f^{-1}[p] \cdot K
$$

$f \mid K$ has closed point inverses. The range of $f \mid K$ is compact and thus $f \mid K$ is subcontinuous. Finally, with the observation that $K$ being compact Hausdorff is regular, we may conclude from Corollary 3.10 that $f \mid K$ is continuous.

However if we choose a net $x_{a}$ in $B \subset K$ such that $x_{a} \rightarrow p$ then $f x_{a}$ is never in the neighborhood $V^{*}$ of $f p$. This contradiction proves the theorem.

The last theorem, 4.4, is a generalization of two theorems of Halfar [3, Ths. 2 and 5]. Halfar's Theorem 5 is the same as our Theorem 4.4 except that Halfar requires $X$ to have property $k_{1}$ at $p$ instead of $k_{2}$ at $p$. Our Example 4.1 shows then that Halfar's Theorem 5 is not as strong as our 4.4 and in particular does not apply to all locally compact spaces.

5. Another characterization of continuity. In this section we give a second characterization of continuity (The first occurred in Theorem 3.4). This characterization was discovered while pursuing the question "How much must the usual net characterization of continuity (see Kelley [4, Th. 1, p. 86]) be relaxed in order that something less than continuity be achieved?" One reasonable answer to 
this question is the subcontinuity concept defined in $\S 2$. The principal theorem of this section seems to suggest this answer.

THEORM 5.1. Let $f: X \rightarrow Y$ be a function where $Y$ is Hausdorff. The following conditions are equivalent:

(a) The function $f$ is continuous.

(b) If $x_{a} \rightarrow p$ in $X$, then there is a $q$ in $Y$ such that $f x_{a} \rightarrow q$.

(c) If $x_{a} \rightarrow p$ in $X$, then there is a subnet of $x_{a}, x_{N b}$, such that $f x_{N b} \rightarrow f p$.

(d) For each $p$ in $X$ there is a $q$ in $Y$ such that $x_{a} \rightarrow p$ implies there is a subnet of $x_{a}, x_{N b}$, such that $f x_{N b} \rightarrow q$.

Note that (b), probably the most interesting equivalence, says that the usual net characterization of continuity is still valid even if it is not required that the image of a convergent net converge to the "right" value. In order to prove that (b) implies (a) we will use the following lemmas which we state separately since they may be of interest in other applications of nets.

LEMMA 5.2. Let $\left(A,>_{a}\right)$ and $\left(B,>_{b}\right)$ be disjoint directed sets which are isomorphic (i.e., there is a one-to-one function $h$ from $A$ onto $B$ such that $\alpha_{1}>_{a} \alpha_{2}$ if and only if $\left.h \alpha_{1}>_{b} h \alpha_{2}\right)$. Then there is $a$ directed set $\left(C,>_{c}\right)$ such that $A$ and $B$ are cofinal subsets of $C$ and $C=A+B$.

Proof. Let $C=A+B$ and define $>_{0}$ as follows: If $\gamma_{1}, \gamma_{2} \in A$ then $\gamma_{1}>{ }_{0} \gamma_{2}$ if and only if $\gamma_{1}>{ }_{a} \gamma_{2}$ and similarly if $\gamma_{1}, \gamma_{2} \in B$. If $\gamma_{1} \in A$ and $\gamma_{2} \in B$ then $\gamma_{1}>_{c} \gamma_{2}$ if and only if $h \gamma_{1}>_{b} \gamma_{2}$ and $\gamma_{2}>_{c} \gamma_{1}$ if and only if $\gamma_{2}>_{b} h \gamma_{1}$. In brief, we order $C$ by leaving the order of $A$ and $B$ the same and identifying points in $A$ with their images in $B$.

It is not difficult to show that $\left(C,>_{c}\right)$ is directed set, and it is clear that $A$ and $B$ are cofinal in $C$.

LEMmA 5.3. Let $X$ be a topological space and $\left\{x_{a}, a \in A\right\}$ and $\left\{z_{b}, b \in B\right\}$ nets in $X$ with disjoint directed sets which are isomorphic. Then there is a net $\left\{w_{c}, c \in C\right\}$ in $X$ such that $x_{a}$ and $z_{b}$ are subnets and $w_{c} \rightarrow p$ if and only if $x_{a} \rightarrow p$ and $z_{b} \rightarrow p$.

Proof. Let $C$ be the directed set constructed as in the previous lemma and define $w_{c}=x_{c}$ if $c \in A, w_{c}=z_{c}$ if $c \in B$. The assertions of the lemma are then clear.

Proof of Theorem 5.1. Since each of conditions (b), (c), and (d) are clearly implied by continuity, we have only to show that each of 
these conditions implies continuity.

Assume condition (b) holds. Let $x_{a} \rightarrow p$. Then $f x_{a} \rightarrow q$ for some $q$ in $Y$. Suppose $q \neq f p$. Let $z_{b}$ be a net which is constantly $p$ and whose directed set is disjoint and isomorphic to that of $x_{a}$. Then by Lemma 5.3 there is a net $w_{c}$ such that $x_{a}$ and $z_{b}$ are subnets and $w_{c} \rightarrow p$. However $f x_{a} \rightarrow q$ and $f z_{b} \rightarrow f p$. Since $Y$ is Hausdorff, $f w_{c}$ cannot converge and we have a contradiction.

Assume condition (d) holds. We will show condition (c) holds. Let $p \in X$ and $q$ be the corresponding point assured by the condition. We wish to show that $q=f p$. But this is evident for if $x_{a}$ is a net constantly $p$ then $x_{a} \rightarrow p$ and thus $f x_{a} \rightarrow f p$. Since $Y$ is Hausdorff $q=f p$.

Finally we show condition (c) implies continuity. Suppose condition (c) holds and $f$ is not continuous. Then there is a $p$ in $X$ and an open neighborhood of $f p, V^{*}$, such that for each neighborhood of $p, U$, there exists a point $x_{U}$ in $U$ such that $f x_{U} \notin V^{*}$. Now the function $x_{U}$ defined on the collection of neigborhoods of $p$ directed downward by inclusion is a net converging to $p$. On the other hand it is clear that for no subnet of $x_{U}, x_{N b}$, is it true that $f x_{N b} \rightarrow f p$. This contradiction completes our proof.

The following corollary has as a corollary to it a theorem announced by Yu-Lee Lee to the effect that if $f$ is a function from uniform space to another which preserves Cauchy nets, then $f$ is continuous.

CoROllaRY TO 5.1 (b) 5.4. Let $f: X \rightarrow Y$ be a function where $(Y, \mathscr{Y})$ is a Hausdorff uniform space. If $f$ maps convergent nets onto Cauchy nets then $f$ is continuous.

Proof. Let $\left(h, Y^{*}, \mathscr{Y}^{*}\right)$ be the Hausdorff completion of $\left(Y, \mathscr{Y}^{\prime}\right)$ where $h$ is a uniform isomorphism of $Y$ into $Y^{*}$. Note $h \circ f$ maps convergent nets onto Cauchy nets. Since $Y^{*}$ is complete, it follows from 5.1(b) that $h \circ f$ is continuous. But then so is $f$ since $h$ is a homeomorphism.

6. Open functions and continuity of their inverses. One feels intuitively that openness of a function should be related to continuity (in some sense) of the inverse function. In this section we find a sense and a setting in which this is indeed the case for the set-valued inverse function.

By an open function, we will mean a function $f: X \rightarrow Y$ such that $U$ is open in $X$ implies $f[U]$ is open in $Y$.

THEOREM 6.2. If $f: X \rightarrow Y$ is a function, the following conditions are equivalent: 
(a) $f$ is open.

(b) $y_{a} \rightarrow q$ implies $f^{-1}[q] \subset \liminf f^{-1}\left[y_{a}\right]$.

(c) $y_{a} \rightarrow q$ implies $f^{-1}[q] \subset \lim \sup f^{-1}\left[y_{a}\right]$.

(See $\S 1$, second paragraph for definitions)

Proof. Assume (a) holds. Let $y_{a} \rightarrow q$ and $p \in f^{-1}[q]$. Suppose $p \notin \liminf f^{-1}\left[y_{a}\right]$. Then there is an open set, $U$, containing $p$ such that frequently $f^{-1}\left[y_{a}\right] \cdot U=\varphi$. Thus $y_{a}$ is frequently outside $f[U]$. But this is absurd since $f[U]$ is a neighborhood of $q$. Thus (b) holds.

Clearly if (b) holds then (c) holds. Hence assume (c) holds. Suppose $f$ is not open. Then there is an open set, $U$, in $X$ and a net $y_{a}$ in $Y-f[U]$ such that $y_{a} \rightarrow q$ for some $q$ in $f[U]$. Thus $f^{-1}[q] \subset \lim$ sup $f^{-1}\left[y_{a}\right]$. Let $p \in f^{-1}[q] \cdot U$. Then $U$ is a neighborhood of $p$ and thus $f^{-1}\left[y_{a}\right] \cdot U$ is frequently nonempty. But this means $y_{a}$ is frequently in $f[U]$ in contradiction to our choice. Therefore (a) holds.

If $E_{a}$ is a net of sets in a topological space $X$ such that lim inf $E_{a}=\lim \sup E_{a}=E$, then we say that the limit of $E_{a}$ exists and we write $\lim E_{a}=E$. With this definition the following theorem follows from the previous theorem and Theorem 1.2.

THEOREM 6.3. The function $f: X \rightarrow Y$ is open and has a closed graph if and only if $y_{a} \rightarrow q$ in $Y$ implies $\lim f^{-1}\left[y_{a}\right]=f^{-1}[q]$.

Proof. By the theorems cited above, $f$ is open and has a closed graph if and only if $y_{a} \rightarrow q$ in $Y$ implies

$$
f^{-1}[q] \subset \liminf f^{-1}\left[y_{a}\right] \subset \lim \sup f^{-1}\left[y_{a}\right] \subset f^{-1}[q] \text {. }
$$

Thus the theorem holds.

A theorem similar to the one immediately preceding is given in Whyburn [10, Th. 4.32, p. 130] for metric spaces.

Now let $X$ be a locally bicompact space (i. e. one which has a basis of open sets with compact closures), and $2^{X}$ be the collection of all nonempty closed subsets of $X$. Consider all sets of the form

$$
\begin{aligned}
& \left\{A \in 2^{X}: A \cdot U_{i}=\varphi \text { and } A \cdot \mathrm{Cl}\left(V_{j}\right)=\varphi\right. \\
& \quad \text { for } i=1, \cdots, n \text { and } j=1, \cdots, m\}
\end{aligned}
$$

where the $U_{i}$ and $V_{j}$ are open and have a compact closure in $X$.

These sets from a basis for a topology for $2^{X}$ which Mrówka calls the $l b c$ topology. Mrówka proves in his paper [9, Th. 4] that for a locally bicompact space $X$, convergence in $2^{X}$ relative to the $l b c$ topology is the same as the convergence of sets previously described (i. e. when $\lim \inf =\lim \sup$ ). Since by Theorem 3.6 if $f: X \rightarrow Y$ has a closed graph then $f^{-1}[y]$ is closed for each $y$, the following theorem follows from the preceding comments and Theorem 6.3. 
6.4 Theorem. Let $f: X \rightarrow Y$ be a function where $X$ is locally bicompact. Let $F$ be the function on $Y$ defined by $F y \equiv f^{-1}[y]$. Let $2^{X}$ have the 1 bc topology. If $f$ is open and has a closed graph then $f: Y \rightarrow 2^{X}$ is continuous. Conversely, if $f$ has closed point inverses and $F: Y \rightarrow 2^{X}$ is continuous then $f$ is open and has a closed graph.

THEOREM 6.5. Let $f$ be an open, continuous function of $X$ onto $Y$ where $X$ is locally bicompact and $Y$ is Hausdorff. Let

$$
\mathscr{D}=\left\{f^{-1}[y]: y \in Y\right\}
$$

and let $\mathscr{D}$ have the relativized 1 be topology. Then $Y$ and $\mathscr{D}$ are topologically equivalent.

Proof. By Theorem 3.4, since $f$ is continuous and $Y$ is Hausdorff, $f$ has a closed graph. Thus by the previous theorem $F: Y \rightarrow D$ defined by $F y=f^{-1}[y]$ is continuous. Thus it remains only to show that $F^{-1}$ is continuous.

Let $f^{-1}\left[y_{a}\right]$ converge to $f^{-1}[q]$ in $\mathscr{D}$. Then

$$
\lim \sup f^{-1}\left[y_{a}\right]=f^{-1}[q] \text {. }
$$

By Lemma 1.1, if $p \in \lim \sup f^{-1}\left[y_{a}\right]$ there is a net $x_{N b}$ in $X$ such that $x_{N b} \rightarrow p$ in $X$ and $f x_{N b}$ is a subnet of $y_{a}$. Now $f$ is continuous and thus $f x_{N b}$ converges to $f p=q$. But

$$
F^{-1}\left(f^{-1}\left[f x_{N b}\right]\right)=f x_{N b} .
$$

Since $f^{-1}\left[f x_{N b}\right]$ is a subnet of $f^{-1}\left[y_{a}\right]$, it follows by Theorem 5.1(c) that $F^{-1}$ is continuous.

Theorems somewhat similar to the previous two theorems may be found in a paper of E. Michael [8] (see in particular Theorem 5.10.2).

\section{REFERENCES}

1. C. Bergé, Espaces Topologiques, Dunod, Paris, 1959.

2. E. Halfar, Compact mappings, Proc. Amer. Math. Soc. 8 (1957), 828-830.

3. - Conditions implying continuity of functions, Proc. Amer. Math. Soc. 11 (1960), 688-691.

4. J. L. Kelley, General Topology, D. Van Nostrand Co., Princeton, 1955.

5. C. Kuratowski, Topologie II, Hafner Publishing Co., New York, 1950.

6. Lee, Yu-Lee, On functions which preserve Cauchy nets, Notices Amer. Math. Soc. 13 (1966), 135.

7. R. Manning, Open and closed transformations, Duke Math. J. 13 (1946), 179-184.

8. E. Michael, Topologies on spaces of subsets, Trans, Amer. Math. Soc. 71 (1951), 152-182.

9. S. Mrowka, On the convergence of nets of sets, Fund. Math. 45 (1958), 237-246. 10. G. T. Whyburn, Analytic topology, Amer. Math. Soc. Colloq. Pub. XXVIII, Providence, Rhode Island, 1942. 
11. Directed families of sets and closedness of functions, Proc. Nat. Acad. Sci. (U.S. A.) 54 (1965), 688-692.

12. - Loosely closed sets and partially continuous functions, Michigan Math. J. 14 (1967), 193-205.

Received August 23, 1967. This paper is taken from the author's doctoral dissertation which was written under the direction of Dr. R. H. Kasriel. The author's graduate study at the Georgia Institute of Technology has been supported by a National Aeronautics and Space Administration Traineeship.

University of North Carolina at Charlotte 



\title{
PACIFIC JOURNAL OF MATHEMATICS
}

\author{
EDITORS
}

\section{H. ROYDEN}

Stanford University

Stanford, California

\author{
J. P. JANS \\ University of Washington \\ Seattle, Washington 98105
}

\section{J. DugundJI}

Department of Mathematics

University of Southern California

Los Angeles, California 90007

\section{RICHARD ARENS}

University of California

Los Angeles, California 90024

\section{ASSOCIATE EDITORS}
E. F. BECKENBACH
B. H. NEUMANN
F. WOLF
K. YOSIDA

\section{SUPPORTING INSTITUTIONS}

\author{
UNIVERSITY OF BRITISH COLUMBIA \\ CALIFORNIA INSTITUTE OF TECHNOLOGY \\ UNIVERSITY OF CALIFORNIA \\ MONTANA STATE UNIVERSITY \\ UNIVERSITY OF NEVADA \\ NEW MEXICO STATE UNIVERSITY \\ OREGON STATE UNIVERSITY \\ UNIVERSITY OF OREGON \\ OSAKA UNIVERSITY \\ UNIVERSITY OF SOUTHERN CALIFORNIA
}

\author{
STANFORD UNIVERSITY \\ UNIVERSITY OF TOKYO \\ UNIVERSITY OF UTAH \\ WASHINGTON STATE UNIVERSITY \\ UNIVERSITY OF WASHINGTON \\ AMERICAN MATHEMATICAL SOCIETY \\ CHEVRON RESEARCH CORPORATION \\ TRW SYSTEMS \\ NAVAL WEAPONS CENTER
}

Mathematical papers intended for publication in the Pacific Journal of Mathematics should be in typed form or offset-reproduced, double spaced with large margins. Underline Greek letters in red, German in green, and script in blue. The first paragraph or two must be capable of being used separately as a synopsis of the entire paper. It should not contain references to the bibliography. Manuscripts, in duplicate if possible, may be sent to any one of the four editors. All other communications to the editors should be addressed to the managing editor, Richard Arens, University of California, Los Angeles, California 90024.

Each author of each article receives 50 reprints free of charge; additional copies may be obtained at cost in multiples of 50 .

The Pacific Journal of Mathematics is published monthly. Effective with Volume 16 the price per volume (3 numbers) is $\$ 8.00$; single issues, $\$ 3.00$. Special price for current issues to individual faculty members of supporting institutions and to individual members of the American Mathematical Society: $\$ 4.00$ per volume; single issues $\$ 1.50$. Back numbers are available.

Subscriptions, orders for back numbers, and changes of address should be sent to Pacific Journal of Mathematics, 103 Highland Boulevard, Berkeley 8, California.

Printed at Kokusai Bunken Insatsusha (International Academic Printing Co., Ltd.), 7-17, Fujimi 2-chome, Chiyoda-ku, Tokyo, Japan.

PUBLISHED BY PACIFIC JOURNAL OF MATHEMATICS, A NON-PROFIT CORPORATION

The Supporting Institutions listed above contribute to the cost of publication of this Journal, but they are not owners of publishers and have no responsibility for its content or policies. 


\section{Pacific Journal of Mathematics}

\section{Vol. 25, No. $3 \quad$ November, 1968}

Philip Marshall Anselone and Theodore Windle Palmer, Collectively

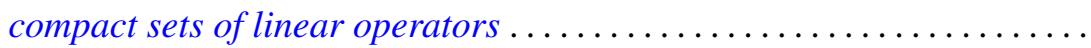

Philip Marshall Anselone and Theodore Windle Palmer, Spectral analysis of collectively compact, strongly convergent operator sequences.........

Edward A. Bender, Characteristic polynomials of symmetric matrices...... 433

Robert Morgan Brooks, The structure space of a commutative locally convex

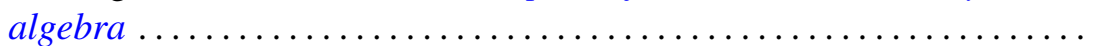

Jacob Feldman and Frederick Paul Greenleaf, Existence of Borel

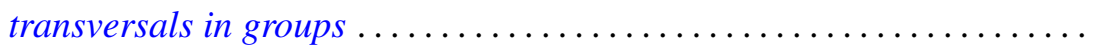

Thomas Muirhead Flett, Mean values of power series 463

Richard Vernon Fuller, Relations among continuous and various

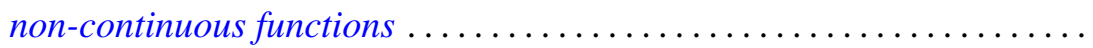

Philip Hartman, Convex sets and the bounded slope condition ............

Marcel Herzog, On finite groups containing a CCT-subgroup with a cyclic Sylow subgroup .

James Secord Howland, On the essential spectrum of Schroedinger

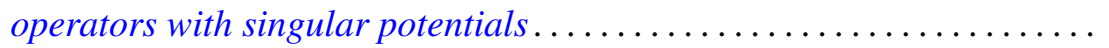

Thomas William Hungerford, On the structure of principal ideal rings .....

Paul Joseph Kelly and Ernst Gabor Straus, Curvature in Hilbert geometries. II mpati Madhusudana Rao, Linear functionals on Orlicz spaces:

Malempati Madhusudana Rao, Linear functionals on Orli
$\quad$ General theory............................
Stanley F. Robinson, Theorems on Brewer sums.......
Ralph Tyrrell Rockafellar, A general correspondence betw

Malempati Madhusudana Rao, Linear functiona
$\quad$ General theory.........................
Stanley F. Robinson, Theorems on Brewer sums
Ralph Tyrrell Rockafellar, A general corresponden

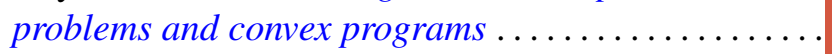

Richard Benjamin Sher, Defining subsets of $E^{3}$ by cubes.

Howard Jacob Weiner, Invariant measures and Cesàro summability... 